\title{
Formazione metodologica, tecnologie didattiche ed esperienze nella promozione delle competenze di insegnamento e apprendimento
}

\author{
Methodologies, educational technologies \\ and experiences in promoting teaching \\ and learning skills
}

\author{
Anna Dipace ${ }^{*}$, Alessia Scarinci ${ }^{* *}$
}

\begin{abstract}
Riassunto
La situazione di emergenza rinveniente dalla diffusione del Covid-19 ha determinato una condizione straordinaria per tutte le istituzioni formative. In questo contributo l'attenzione è focalizzata in modo particolare sull'università che ha visto migliaia di docenti improvvisamente alle prese con la necessità di adottare soluzioni di didattica a distanza per i propri studenti pur non avendo specifiche competenze ed esperienze maturate in questo ambito. Questa situazione ha messo in evidenza una serie di bisogni collegati ad una specifica domanda di formazione che interessa tanto gli aspetti formativi quanto quelli di ricerca didattica che sta cercando di fornire una lettura del fenomeno al fine di alimentare una riflessione matura sul rapporto tra media, ambienti (reali o virtuali) di apprendimento e strategie di insegnamento. Tuttavia, da questa situazione di emergenza possono derivare situazioni inedite di riprogettazione qualificata della formazione superiore innanzitutto attraverso un aggiornamento della didattica universitaria e, dunque, di specifiche competenze dei propri docenti in grado di utilizzare strategie, metodi e strumenti (sia in presenza che a distanza) per favorire l'apprendimento attivo, costruttivo e interattivo. Il contributo, partendo dal framework di innovazione nell'alta formazione proposto da Gilly Sal-
\end{abstract}

\footnotetext{
* Università degli Studi di Modena-Reggio Emilia.

** Università degli Studi di Bari.

Le due Autrici hanno condiviso l'ideazione, la progettazione e lo sviluppo del saggio. Nella stesura del testo, i $\S$ Introduzione, 1 e 2 sono stati curati da Anna Dipace e i $\S 3$ e 4 e le conclusioni sono stati curati da Alessia Scarinci.
}

Excellence and Innovation in Learning and Teaching (ISSNe 2499-507X), Special Issue

Doi: $10.3280 /$ exioa0-2021oa11130 
mon, presenta un'analisi esplorativa sui MOOC offerti da alcune delle principali piattaforme a livello internazionale come opportunità di formazione metodologica per i docenti durante il periodo più difficile dell'emergenza Covid-19.

Parole Chiave: MOOC, Università, strategie di insegnamento, tecnologie educative, Covid-19

\begin{abstract}
The emergency situation arising from the spread of Covid-19 has determined an extraordinary condition for all educational institutions. In this paper, the focus is specifically on the university which has seen thousands of teachers suddenly grappling with the need to adopt distance learning solutions for their students despite not having specific skills and experience in this area. This situation has highlighted a series of needs linked to a specific training demand that affects both the educational aspects and those of didactic research which is trying to provide a reading of the phenomenon in order to feed a mature reflection on the relationship between media, environments (real or virtual) learning and teaching strategies. However, this emergency situation may result in unprecedented situations of qualified redesign of higher education, first of all through a requalification of university teaching and, therefore, of specific skills of their teachers able to use strategies, methods and tools (both in presence and at a distance) to foster active, constructive and interactive learning. The paper, starting from the framework of innovation in higher education proposed by Gilly Salmon, describes an exploratory analysis on the MOOCs offered by some of the main international platforms as a methodological training opportunity for teachers during the most difficult period of the Covid-19.
\end{abstract}

Keywords: MOOC, University, teaching strategies, educational technology, Covid-19.

\title{
Introduzione
}

Gli sviluppi nel settore della tecnologia si stanno rivelando molto più rapidi rispetto a quelli della ricerca didattica e quindi, mentre la tecnologia offre ampie opportunità per migliorare $\mathrm{i}$ risultati educativi, i tentativi di sfruttare la tecnologia per l'apprendimento potrebbero fallire se non sono basati sulla scienza dell'apprendimento. L'emergenza Covid-19 ha messo ancora più in evidenza 
alcune lacune nell'ambito della formazione metodologica in particolare collegata alle tecnologie didattiche dei docenti universitari. La necessità di rispondere alla sfida di accogliere i bisogni di innovazione didattica è sentita da una parte consistente dell'educazione formale. La pandemia del Covid-19 ha radicalmente modificato, a livello globale, i sistemi educativi, dall'infanzia all'alta formazione. I dati che l'Unesco ha messo a disposizione online dimostrano che, al 13.04.20, il 90,1\% degli studenti a livello mondiale sono stati interessati dalla chiusura delle istituzioni formative (Figura 1).

Fig. 1 - Dati Unesco sul monitoraggio delle chiusure delle scuole nel periodo emergenza Covid-19. Situazione al 13.04.20
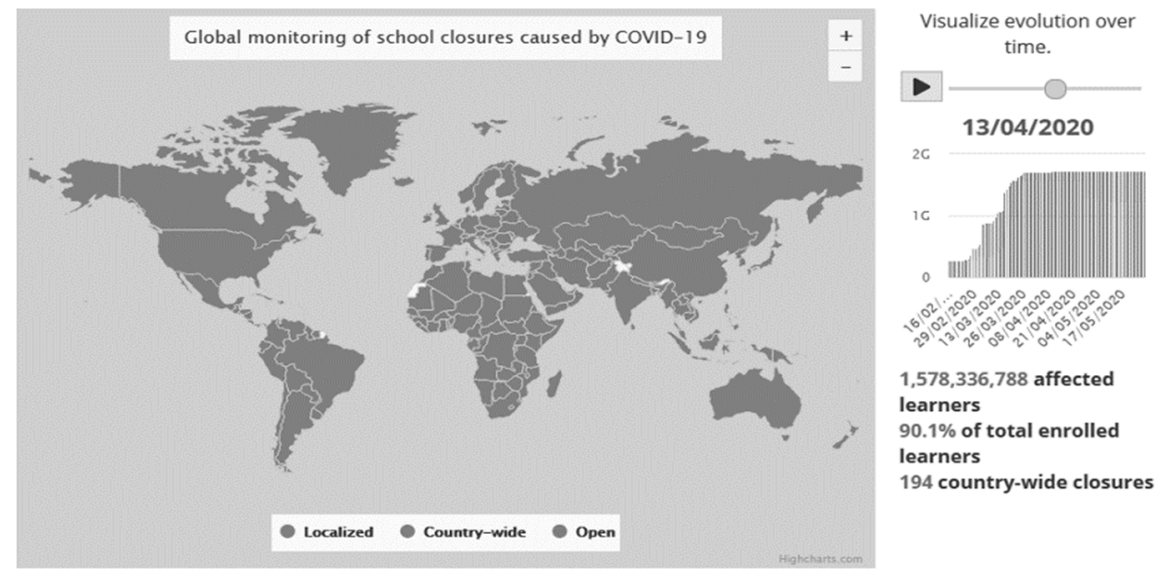

Fonte: https://en.unesco.org/covid19/educationresponse

L'immagine (Fig. 1) mette in evidenza questa situazione di emergenza e dimostra che, in modo del tutto eccezionale, un numero considerevole di istituzioni hanno dovuto pensare come garantire il diritto all'educazione e formazione nonostante la chiusura fisica delle scuole e delle università.

In moltissimi casi, le istituzioni hanno cercato di far fronte a tale emergenza spostando la didattica online. Inevitabilmente, questo passaggio ha richiesto un ripensamento di tutto l'assetto organizzativo e, quindi, uno sforzo notevole da parte di tutti gli attori e le istituzioni coinvolte. In particolare, perché si realizzasse questo passaggio dagli spazi fisici agli ambienti virtuali, è stato necessario mettere in atto una sostanziale rivisitazione dei contesti, dei linguaggi, dei codici comunicativi, delle metodologie, degli strumenti e delle relazioni. Tutti, dai docenti agli studenti, dalle istituzioni alle famiglie, hanno dovuto adattarsi 
e integrarsi in una riformulazione e rimodulazione della propria quotidianità per affrontare l'emergenza, provando a rispondere alle nuove condizioni sociali, culturali e organizzative.

In questo contributo ci soffermeremo sulla figura del docente, con uno sguardo specifico sulla disponibilità di percorsi formativi volti alla preparazione metodologica necessaria a far fronte ad una serie di esigenze, collegate alle tecnologie dell'istruzione come anche legate all'emergenza Covid-19.

\section{Università tra formazione e investimenti}

In generale, le università hanno bisogno di mettere in atto una serie di investimenti su più livelli, che vanno dall'attivazione di processi di ricerca e di formazione dei docenti, volti allo sviluppo di competenze per un uso strategico delle tecnologie didattiche, alla predisposizione di risorse strutturali e infrastrutturali (anche tecnologiche), alla digitalizzazione dei servizi e dei sistemi degli atenei.

Insegnare online richiede l'utilizzo di diversi approcci pedagogici rispetto alla lezione tradizionale. Per tali ragioni, è importante che i docenti siano adeguatamente formati per essere in grado di agire nei diversi ambienti che vanno dalla centralità della presenza alla centralità della distanza.

I repentini mutamenti culturali, sociali, economici e tecnologici stanno via via allontanandosi dall'idea di tradizione, che perde rilevanza a favore di una tendenza che mette in ombra il valore del progresso e fa luce sull'idea dell'innovazione. Le tecnologie e i media digitali sembrano essere gli strumenti più idonei per rientrare nello scenario dell'innovazione, tanto che, come sostiene Marco Gui $(2019$, p. 27) «innovazione e tecnologia digitale si sovrappongono sempre di più». Quello che adesso sicuramente abbiamo imparato è che la tecnologia da sola non può trasformare i paradigmi educativi, il modo di insegnare e di apprendere. Se, da un punto di vista più generale, tutto può cambiare utilizzando le tecnologie nella didattica, dal punto di vista pedagogico potrebbe non cambiare nulla.

Il profilo dell'insegnante (e le sue competenze) è cambiato. Il docente oggi è un ricercatore, uno studioso e un intellettuale capace di progettare percorsi didattici e ambienti di apprendimento e di valutarne l'efficacia (Scarinci \& Dipace, 2019).

Il nuovo profilo del docente è caratterizzato dall'avere una molteplicità di prospettive: da docente come progettista, capace di progettare percorsi didattici 
ed esperienze di apprendimento in ambienti misti, reali e virtuali (Laurillard, 2013), a docente come moderatore, facilitatore.

Nikendei e colleghi (2016) hanno condotto una interessante indagine su un campione di oltre duemila docenti universitari a cui è stato chiesto di definirsi, identificando sei descrittori corrispondenti ai ruoli di: facilitatore, fornitore di informazioni, pianificatore di curricula, valutatore e creatore di strumenti di verifica, modello professionale e sviluppatore di risorse. Harden, a partire da tali risultati, ha delineato gli otto ruoli del docente universitario, definendolo: fornitore di informazioni e coach; facilitatore e mentore; progettista di curriculum (pianificatore e realizzatore); valutatore e diagnostico; role model come docente e professionista; manager e leader; studioso e ricercatore; professionista (Harden \& Lilley, 2018).

Questa connotazione professionale così poliedrica del docente fa sì che oggi diventi indispensabile non soltanto sapere, essere esperti, ma anche possedere competenze strategiche di comunicazione didattica. Saper progettare una lezione, delineare dettagliatamente un syllabus, implica anche saper articolare un intervento didattico che si avvalga di nuovi linguaggi, paradigmi, strumenti, ambienti digitali, contesti e media che contribuiscono a dare forma all'identità e alle competenze dei soggetti in formazione. Si tratta di modelli pedagogici che richiedono l'uso di ambienti di apprendimento nei quali è necessaria una interazione comunicativa che si realizza tra pari e tra pari e formatori a diverso titolo (insegnanti, esperti, facilitatori, counselor, ecc.) e che può essere agevolata dall'inserimento dei linguaggi multimediali e multimodali negli ambienti formativi. Tali linguaggi e relativi strumenti e tecnologie si connotano come "partner cognitivi" e supportano la creazione di una interdipendenza tra saperi formali, informali e non formali (Limone, Dipace \& Martiniello, 2016).

\section{Tecnologie e innovazione didattica nei contesti universitari}

Il ritmo dell'innovazione per l'apprendimento e l'insegnamento sta notevolmente accelerando per far fronte ad una serie di forze dirompenti e costruttive che si stanno insinuando nell'ambito dell'alta formazione, sfidando i modelli didattici e comunicativi tradizionali (Christensen \& Horn, 2013; Miller et al., 2014). L'adozione delle tecnologie emergenti, e l'applicazione di forme di innovazione didattica in ambito universitario, offrono sicuramente una serie di straordinarie opportunità che inevitabilmente vanno a stravolgere taluni equilibri e assetti che in alcuni (molti) casi possono sembrare resistenti al cambiamento. 
Questo frequente ancoraggio alla tradizione e resistenza al cambiamento, in molti casi, può comportare l'adozione più lenta delle pratiche di innovazione con conseguente rallentamento dei processi. Il conservatorismo di alcune istituzioni formative, in particolare le università, è piuttosto noto, ma questo non può rappresentare ancora oggi un ostacolo per la messa in atto di azioni che investono nel miglioramento delle pratiche e, quindi, nella formazione dei docenti. Le tecnologie possono essere un valido supporto tanto per l'innovazione didattica con gli studenti, quando per facilitare l'accesso alla formazione continua e all'aggiornamento professionale dei docenti, in uno scenario orientato al faculty development. La sfida a cui sono chiamate le università oggi è quella di essere in grado di promuovere la crescita e migliorare l'esperienza didattica, con ricadute sui risultati di apprendimento. Le istituzioni formative stanno cercando di rispondere a tali esigenze organizzando una serie di attività, nell'ambito dei programmi e delle azioni di faculty development, volte a coinvolgere attivamente i docenti perché possano acquisire le competenze necessarie e disporre di strumenti efficaci per una didattica di qualità. L'università tra innovazione e cambiamento è un tema al centro di diverse ricerche in ambito internazionale. Una proposta per far fronte all'innovazione in ambito accademico è stata messa a punto da Gilly Salmon (2014). Si tratta del Framework for innovation, un modello di trasformazione delle pratiche rivolto prevalentemente ai contesti accademici e quindi all'alta formazione. Questo framework parte dalla possibile corrispondenza che l'università può individuare tra le sue risorse, strutture e competenze interne e le opportunità e rischi che provengono dall'ambiente esterno. In questo modo si possono valorizzare le capacità e i punti di forza di un'istituzione, evidenziando quindi in cosa eccelle, e si può capire quali strategie utilizzare per renderla competitiva e quindi cosa può fare meglio e in modo diverso.

Il framework proposto da Salmon (2014) delinea due vie per l'innovazione, una moderata e una radicale, e a tal fine suggerisce un modello a 4 quadranti per la trasformazione nei contesti di alta formazione.

Per ottenere i migliori risultati centrati sullo studente, Salmon propone un passaggio graduale attraverso quattro fasi che guidano la trasformazione dell'istruzione superiore sulla base di due elementi, che pone sui due assi e che indicano le esigenze di mercato (i contesti economici) e la progettazione didattica e le tecnologie.

Il framework colloca nel quadrante più alto i cambiamenti radicali attraverso cui è possibile mettere in atto una vera e propria trasformazione dell'istruzione superiore. Perché questa trasformazione avvenga, sono necessarie pratiche aggiornate, pedagogicamente innovative, che considerino le sfide già esistenti poste 
dalla nuova era tecnologica in modo olistico. L'immagine del framework (Figura 2) illustra questa suddivisione in quadranti e indica per ognuno il tempo necessario perché possa considerarsi risolto e quindi si possa passare al successivo:

- Quadrante 1: fase in cui le istituzioni fanno riferimento alle tecnologie di cui dispongono già, e le utilizzano al proprio interno per risolvere i problemi e per migliorare la qualità della didattica. L'investimento è sulla progettazione didattica, sullo sviluppo professionale e sul supporto del personale, e quindi su un processo di ripensamento delle pratiche e degli spazi mettendo in atto l'innovazione in modo molto graduale, ma comunque in grado di produrre un impatto diretto sull'apprendimento degli studenti. Il livello di rischio è basso e la tempistica per la realizzazione va da uno a due anni.

- Quadrante 2: fase in cui le istituzioni accettano la sfida di un approccio diverso che, partendo dai punti di forza del quadrante 1 , si muove verso nuove missioni e nuovi fronti. Un esempio può essere il passaggio dalla didattica in presenza a quella blended o interamente online. In questo caso, l'università prevede un investimento anche sullo sviluppo organizzativo. Il livello di rischio è legato alla presenza di alcuni possibili investimenti, e alla scelta dei possibili partner con cui collaborare per la realizzazione. La tempistica da prevedere per la sua realizzazione va tipicamente da un anno ad un anno e mezzo.

- Quadrante 3: fase in cui si fanno investimenti sulle nuove tecnologie disponibili sul mercato. Si considera la possibilità di implementare e integrare tali tecnologie nell'ambito della didattica universitaria, comprendendo anche i social media e altri dispositivi come i MOOC o le Open Educational Resources. L'idea è quella di realizzare dei prototipi su piccola scala che possano rispondere alla natura mutevole dell'apprendimento e alle molteplici esigenze di insegnamento. Il livello di rischio è medio-alto poiché vanno considerate alcune minacce legate alla prototipazione. Va considerato un tempo di realizzazione che va da uno a due anni.

- Quadrante 4: fase più impegnativa e rischiosa, ma anche potenzialmente più gratificante. Perché si realizzi, è necessaria una visione più radicale del cambiamento, che richiede il ricorso ad investimenti importanti e quindi un approccio orientato a guardare oltre l'orizzonte. In questo modo, si possono fare investimenti in progetti che esplorano nuove idee, nuovi approcci e vanno a testare nuove tecnologie emergenti, come i dispositivi indossabili, la realtà virtuale e aumentata, la gamification, ecc. Inoltre, si richiede la disponibilità a stabilire partnership, a sperimentare nuovi prodotti, nuovi mercati e avviare nuove missioni. Il livello di rischio è piuttosto alto, perché 
richiede un investimento considerevole. I tempi di realizzazione sono un po' più lunghi e vanno da $2 \mathrm{a} 4$ anni.

Fig. 2 - Framework trasformativo per l'innovazione dell'apprendimento

Framework trasformativo per l'innovazione dell'apprendimento

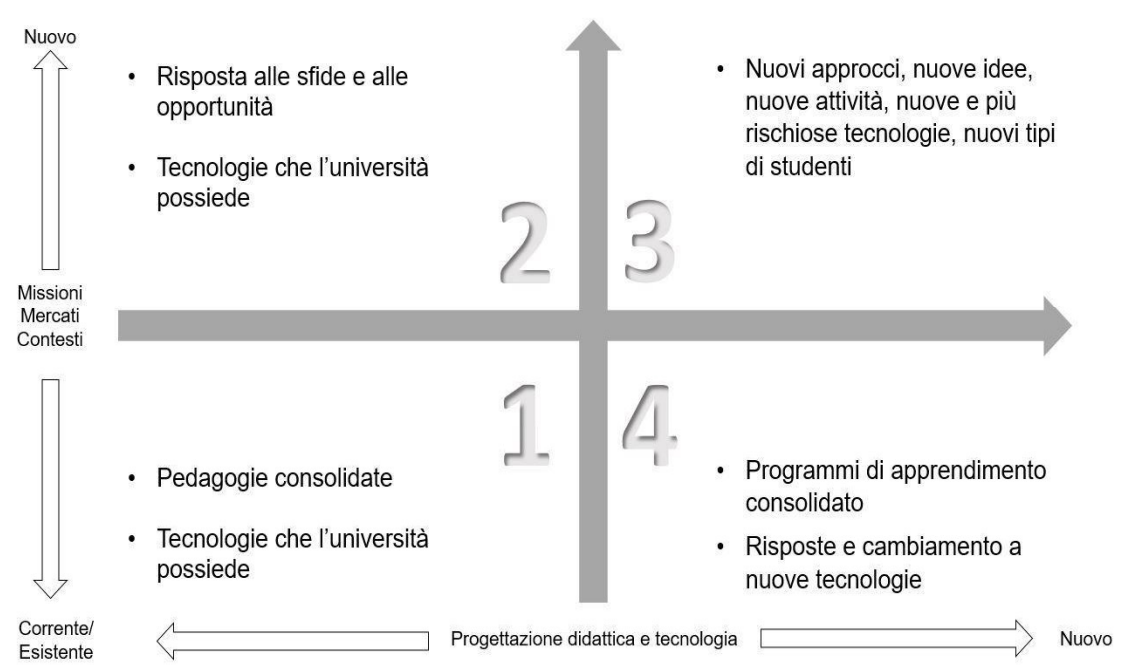

Fonte: tratto e tradotto da Salmon, 2014

\section{Innovazione e tecnologie didattiche: l'impatto e la risposta all'emergenza Covid-19}

Quanto detto finora dimostra che, perché si possano mettere in atto dei cambiamenti capaci di produrre un impatto positivo sull'organizzazione, sono necessari una serie di investimenti economici e strumentali, e soprattutto è necessario prevedere dei tempi di realizzazione adeguati, che permettano di stabilire e ristabilire i giusti equilibri nonostante le innovazioni. Il framework descritto nel paragrafo precedente dimostra come sia necessario dedicare il giusto tempo ad ogni azione innovativa perché questa possa essere implementata, integrata e quindi accettata e apprezzata dagli attori e dalle istituzioni coinvolte. Quanto è accaduto durante l'emergenza sanitaria legata al Covid-19 ha imposto alle istituzioni educative un repentino e radicale cambiamento, senza tener conto di tutta una serie di attività preparatorie che invece sono previste in situazioni di 
normalità. Gli effetti di questa radicale e improvvisa trasformazione sono stati molteplici e hanno fatto emergere le grosse lacune delle università sotto diversi profili, come la carenza e inadeguatezza degli strumenti e delle infrastrutture tecnologiche, la carenza di una flessibilità organizzativa, il bisogno di competenze didattiche dei docenti nell'ambito delle tecnologie, un deficit di competenze digitali dei docenti e anche degli studenti.

Tali lacune hanno fatto sì che il tipo di didattica che è stata realizzata è stata definita Emergency Remote Teaching, in contrapposizione all'Effective Online Learning (Hodges, Moore, Lockee, Trust, \& Bond, 2020). Esperienze di insegnamento-apprendimento online ben pianificate sono significativamente diverse dai corsi online di emergenza che invece sono stati messi in atto in modo rapido e senza una opportuna progettazione preliminare.

L'Effective Online Learning è il risultato della combinazione tra diverse dimensioni e modelli pedagogici, che richiedono competenze specifiche di progettazione didattica anche nell'ambito delle tecnologie dell'istruzione e degli ambienti di apprendimento.

Tutto questo impatta sulla qualità dell'istruzione offerta e quindi sull'apprendimento degli studenti.

Questi elementi non sono stati considerati nella maggior parte dell'offerta didattica durante l'emergenza Covid-19, e infatti, in risposta ai bisogni formativi dei docenti, degli studenti e di tutti gli attori coinvolti, sono state mobilitate una serie di forze e di azioni:

- a livello locale (università, case editrici, organizzazioni, società scientifiche);

- a livello nazionale (Ministero, Indire, Anvur, ecc.);

- a livello europeo (European Commission, EHEA, EUA, associazioni, ecc.);

- a livello mondiale (Unesco, provider di Mooc, associazioni, ecc.)

In questo contesto, ci soffermiamo in modo particolare sui Mooc che sono stati messi a disposizione sui vari provider nazionali e internazionali relativamente ai temi della didattica con le nuove tecnologie per rispondere ai bisogni di formazione metodologica dei docenti in un momento così particolare. 


\section{I Mooc per offrire ai docenti opportunità di formazione metodo- logica: un'analisi esplorativa}

I Mooc rappresentano la nuova offerta di percorsi formativi messi a disposizione attraverso la rete, generalmente di livello universitario e con accesso gratuito, almeno di base. I Mooc nascono grazie all'intuizione di alcune università prestigiose come Harvard, Princeton e Stanford, e, dal 2011 ad oggi, hanno riscontrato un incredibile successo proprio perché permettono a chiunque di accedere a contenuti formativi attendibili e di alto livello, senza richiedere alcun prerequisito e senza alcun limite di accesso. A testimoniare il successo riscosso dai Mooc sono le numerose piattaforme disponibili ad oggi sul mercato nazionale e internazionale. Coursera rientra tra le prime piattaforme rese disponibili sul web, fondata da alcuni docenti dell'Università di Stanford, in collaborazione con la Princeton University, l'Università del Michigan, e l'Università della Pennsylvania. Dopo questa iniziativa pioneristica, diversi portali hanno investito nei Mooc: tra questi, si ricorda FutureLearn, nato in Gran Bretagna in collaborazione con la Open University che rappresenta la piattaforma MOOC più estesa del Regno Unito, composta da 54 università britanniche e internazionali.

La semplice analisi che qui si presenta ha preso in considerazione le due piattaforme appena citate, Coursera e FutureLearn, e ha provato ad individuare i Mooc disponibili rivolti prevalentemente ad un pubblico interessato a sviluppare competenze nella progettazione didattica in ambienti di apprendimento online.

La scelta è ricaduta sulle due piattaforme citate anche perché sono quelle che presentano maggiori strumenti di ricerca avanzata, permettendo di fare una serie di analisi che si presentano nel dettaglio qui di seguito.

La ricerca di corsi, su entrambe le piattaforme, ha utilizzato i due seguenti tag: "Teach online" e "Learn online".

Dall'analisi dei primi 100 corsi che la ricerca per tag ha restituito, sono stati selezionati i 10 corsi per ciascuna piattaforma con i titoli più attinenti e rilevanti (sono emersi corsi che, pur riportando i due tag, non sono in linea con il tema che si sta analizzando in questo contesto).

La Tabella 1 mostra i titoli, in ordine alfabetico, dei Mooc individuati per le due piattaforme. 
Tab. 1 - Titoli dei Mooc selezionati suddivisi per Provider

\begin{tabular}{cc}
\hline \multicolumn{1}{c}{ Coursera } & FutureLearn \\
\hline $\begin{array}{c}\text { Blended Language Learning: Design and Practice } \\
\text { for Teachers }\end{array}$ & $\begin{array}{c}\text { An Introduction to Computer Networking for } \\
\text { Teachers }\end{array}$ \\
\hline $\begin{array}{c}\text { Blended Learning: Personalizing Education for } \\
\text { Students }\end{array}$ & $\begin{array}{c}\text { Becoming a Better Teacher: Exploring Professional } \\
\text { Development }\end{array}$ \\
$\begin{array}{c}\text { e-Learning Ecologies: Innovative Approaches to } \\
\text { Teaching and Learning for the Digital Age }\end{array}$ & How to Learn Online: Getting Started \\
$\begin{array}{c}\text { Foundations of Teaching for Learning: Curriculum } \\
\text { Foundations of Virtual Instruction }\end{array}$ & How To Teach Online: Providing Continuity for \\
Students
\end{tabular}

Per i suddetti Mooc, utilizzando gli strumenti messi liberamente a disposizione dalle due piattaforme, sono state effettuate alcune semplici rilevazioni di dati per cercare di fare una analisi più attenta di questi corsi selezionati.

In particolare, i dati a disposizione hanno permesso di analizzare:

- numero di iscritti;

- durata complessiva e parziale;

- tipologia e numero di attività previste;

- impatto della formazione sullo sviluppo professionale;

- livello di difficoltà del corso (a chi è rivolto il corso). 


\subsection{Analisi dei dati}

Tab. 2 - Analisi numero di utenti per provider Mooc

\begin{tabular}{lccccc}
\hline Provider & N. corsi & Totale & Media & Minimo & Massimo \\
\hline Futurelearn & 10 & 356.737 & $35.673,70$ & 1.805 & 94.212 \\
Coursera & 10 & 230.240 & $23.024,00$ & 4.652 & 230.240 \\
Totali & 20 & 586.977 & $29.348,85$ & & \\
\hline
\end{tabular}

L'analisi del totale degli utenti (Tabella 2) che risultano iscritti ai corsi ha permesso di calcolare il numero medio di utenti per corso, il numero minimo di utenti per corso ed il numero massimo di utenti per corso. Nell'ambito dei Mooc, il numero di iscritti non coincide con il numero di reali partecipanti alle attività formative, giacché proprio perché si tratta di corsi gratuiti senza frequenza obbligatoria, a meno che non si scelga l'opzione che rilascia certificati e crediti (normalmente a pagamento); questo incide sull'elevato tasso di abbandono (Fei \& Yeung, 2015; Xing, Chen, Stein, \& Marcinkowski, 2016; MorenoMarcos, Muñoz-Merino, Maldonado-Mahauad, Pérez-Sanagustín, AlarioHoyos, \& Kloos, 2020). Il numero di iscritti fa comunque comprendere in larga parte l'interesse che un Mooc suscita nel pubblico di riferimento.

Tab. 3 - Analisi delle frequenze percentuali della durata in settimane dei corsi

\begin{tabular}{lcccc}
\hline Provider & Sino a 2 & da 2 a 4 & da 5 a 8 & oltre le 8 \\
\hline Futurelearn & $20,0 \%$ & $80,0 \%$ & $0,0 \%$ & $0,0 \%$ \\
Coursera & $0,0 \%$ & $40,0 \%$ & $50,0 \%$ & $10,0 \%$
\end{tabular}

La Tabella 3 mostra le durate medie dei corsi in settimane, e dall'analisi dei dati emerge che i corsi disponibili sul portale FutureLearn sono stati progettati prevedendo un impegno temporale minore e quindi sono più brevi.

Tab. 4 - Analisi delle frequenze percentuali dell'impegno settimanale medio in ore

\begin{tabular}{lccc}
\hline Provider & sino 2 & da 2 a 3,5 & da 3,5 a 5,5 \\
\hline Futurelearn & $50,0 \%$ & $40,0 \%$ & $10,0 \%$ \\
\hline Coursera & $30,0 \%$ & $30,0 \%$ & $40,0 \%$
\end{tabular}

L'analisi condotta (Tabella 4) mette in evidenza una tendenza per tutti e due i portali a progettare corsi che richiedono un impegno medio settimanale non 
superiore alle 3,5 ore (il $75 \%$ dei corsi ha una durata massima non superiore alle 3,5 ore). I corsi che richiedono un impegno maggiore sembrano comunque riscontrare un certo interesse, infatti la Tabella 5 che segue indica che il $49.6 \%$ degli utenti si iscrivono a corsi con impegno settimanale superiore alle 2 ore ma inferiore alle 3,5 . Solamente $1 ' 11,5 \%$ si iscrive a corsi con impegno settimanale superiore alle 3.5 ore. Ovviamente, sulla base di quanto già detto, non è possibile conoscere il dato di quanti iscritti hanno portato a termine il corso e quindi non è possibile immaginare se la durata incida al momento in cui ci si decide di effettuare l'iscrizione.

Tab. 5 - Distribuzione percentuale degli utenti dei corsi rispetto all'impegno settimanale medio in ore

\begin{tabular}{cccc}
\hline Ore & Futurelearn & Coursera & Totale \\
\hline sino 2 & $54,5 \%$ & $14,7 \%$ & $38,9 \%$ \\
\hline da 2 a 3,5 & $40,9 \%$ & $63,1 \%$ & $49,6 \%$ \\
da 3,5 a 5,5 & $4,6 \%$ & $22,2 \%$ & $11,5 \%$ \\
Totale & $100,0 \%$ & $100,0 \%$ & $100,0 \%$ \\
\hline
\end{tabular}

Tab. 6-Distribuzione percentuale degli utenti dei corsi rispetto alla durata media settimanale dei corsi

\begin{tabular}{cccc}
\hline Settimane & Futurelearn & Coursera & Totale \\
\hline Sino a 2 & $14,1 \%$ & $0,0 \%$ & $8,6 \%$ \\
\hline da 2 a 4 & $85,9 \%$ & $22,2 \%$ & $60,9 \%$ \\
da 5 a 8 & $0,0 \%$ & $73,2 \%$ & $28,7 \%$ \\
oltre le 8 & $0,0 \%$ & $4,6 \%$ & $1,8 \%$ \\
\hline Totale & $100,0 \%$ & $100,0 \%$ & $100,0 \%$ \\
\hline
\end{tabular}

L'impegno totale in termini di settimane (Tabella 6) necessarie per il completamento del corso sembra concentrarsi in un trend che va da 2 a 4 settimane, quindi meno di un mese, se si mantiene un ritmo costante. Questo dato è interessante perché comunica l'impegno standard che si potrebbe considerare nella progettazione di corsi sulle metodologie e tecnologie didattiche.

Coursera permette di rilevare i risultati in termini di ricadute professionali da parte degli utenti attraverso la somministrazione di questionari a cui gli stessi rispondono a completamento del corso. Si individuano tre categorie principali: 
- iniziare una nuova carriera;

- ricevere un beneficio tangibile nella propria carriera;

- ricevere una promozione in termini economici.

Una buona percentuale di utenti (Tabella 7) sembra rientrare nella prima categoria, il 17,5\% nella seconda e comunque un dato interessante è anche quello relativo al quasi $10 \%$ che riceve benefici economici.

Tab. 7 - Grado di rilevanza dei corsi di Coursera nella carriera professionale

\begin{tabular}{lcccccc}
\hline & $\begin{array}{c}\text { Dato non } \\
\text { disponibile }\end{array}$ & sino a $33 \%$ Jal $34 \%$ a $45 \%$ Ja & $\mathbf{4 6 \%}$ a $50 \%$ & oltre $50 \%$ & Media \\
\hline $\begin{array}{l}\text { started a new career } \\
\text { after completing these } \\
\text { courses }\end{array}$ & $50,0 \%$ & $10,0 \%$ & $10,0 \%$ & $10,0 \%$ & $20,0 \%$ & $12,5 \%$ \\
$\begin{array}{l}\text { got a tangible career } \\
\text { benefit from this course }\end{array}$ & $30,0 \%$ & $50,0 \%$ & $10,0 \%$ & $10,0 \%$ & $0,0 \%$ & $17,5 \%$ \\
$\begin{array}{l}\text { got a pay increase or } \\
\text { promotion }\end{array}$ & $60,0 \%$ & $30,0 \%$ & $0,0 \%$ & $10,0 \%$ & $0,0 \%$ & $10,0 \%$ \\
\hline
\end{tabular}

Altri dati sono disponibili dall'analisi dei due portali e possono essere utili per comprendere i bisogni formativi degli utenti interessati a formarsi nell'ambito delle metodologie didattiche con le nuove tecnologie e in ambienti di apprendimento online.

Tab. 8 - Analisi delle frequenze percentuali del livello dei corsi

\begin{tabular}{lcccc}
\hline Provider & $\begin{array}{c}\text { Non } \\
\text { disponibile }\end{array}$ & Beginner & Intermediate & Mixed \\
\hline Futurelearn & $90,0 \%$ & $10,0 \%$ & $0,0 \%$ & $0,0 \%$ \\
Coursera & $10,0 \%$ & $60,0 \%$ & $10,0 \%$ & $20,0 \%$ \\
Totali & $50,0 \%$ & $35,0 \%$ & $5,0 \%$ & $10,0 \%$ \\
\hline
\end{tabular}

Le piattaforme FutureLearn e Coursera distinguono i corsi in base alla difficoltà/complessità (Tabella 8), e quindi al livello di expertise richiesto ai partecipanti, e prevedono tre categorie: beginner, intermediate e mixed. Una buona parte dell'offerta formativa $(35,0 \%)$ è rivolta alla prima categoria, mettendo in 
evidenza i bisogni di formazione di base degli utenti interessati a questa tipologia di corsi.

La raccolta dei dati è stata portata a termine il 10-8-2020 e, a distanza di poche settimane, sono stati pubblicati altri corsi sul "teach online" e "learn online". Questo dimostra un certo interesse sia in termini di offerta che di domanda e quindi mette l'accento sull'urgenza di rispondere in modo efficace attraverso più strategie e strumenti.

\section{Conclusioni}

La storia insegna che una serie di eventi, come le pandemie, le guerre, i terremoti, i disastri, possono interferire con i processi di insegnamento e apprendimento e stravolgerne gli equilibri, in modo anche sostanziale, come è accaduto per l'emergenza Covid-19 in corso da diversi mesi. Un recente studio svolto in Nuova Zelanda (Dohaney, De Roiste, Salmon \& Sutherland, 2020) ha messo in evidenza quanto accade a livello universitario in occasione di queste situazioni emergenziali e ha individuato nella resilienza un possibile fattore di successo per il superamento della crisi.

Nelle università, gli elementi fondamentali per la costruzione della resilienza sono il supporto, la comunità, la leadership e la pianificazione. Un elemento interessante della ricerca svolta sta nella profilazione degli accademici resilienti e delle istituzioni resilienti. In particolare, tra le qualità di un accademico resiliente, oltre alla flessibilità, alla capacità di adattamento, alla disponibilità alla collaborazione, vi è, a livello delle capacità, l'essere digitalmente competenti, ben organizzati e disporre di un buon pensiero creativo. Le competenze metodologiche e digitali rientrano quindi tra le caratteristiche principali per far fronte a situazioni di emergenza e quindi la possibilità di accedere a corsi, anche aperti, massivi e online, può rappresentare un grosso vantaggio per una formazione di qualità su temi di particolare interesse. Inoltre, tra le caratteristiche delle istituzioni resilienti vi è la capacità di promuovere strategie di apprendimento flessibili, miste e digitali e il supporto allo staff per lo sviluppo dell'alfabetizzazione digitale.

Allo stesso modo, si individuano una serie di barriere che ostacolano lo sviluppo della resilienza e tra queste vi è la mancanza di competenze digitali del personale docente e amministrativo e degli studenti, la carenza di sistemi digi- 
tali per far fronte alle emergenze e quindi ai passaggi da una didattica in presenza ad una totalmente a distanza, la mancanza di flessibilità e di predisposizione al cambiamento da parte degli accademici.

La riorganizzazione improvvisa e radicale che le università hanno messo in atto per rispondere all'emergenza Covid-19 ha interessato non solo gli spazi, ma anche gli strumenti, i metodi e anche i ruoli. Infatti, i docenti si sono trovati a gestire stress e carichi di lavoro notevoli per riorganizzare le lezioni a distanza, spesso senza un adeguato supporto metodologico e anche tecnico (Hodges et al. 2020). Particolarmente importante, anche per la riduzione del carico di lavoro e di stress, è la conoscenza approfondita di metodologie che permettano loro di ripensare i propri insegnamenti in modo adeguato al fine di progettare e facilitare esperienze di apprendimento online significative.

\section{Bibliografia}

Christensen, C.M., \& Horn, M.B. (2013). How disruption can help colleges thrive. The Chronicle of Higher Education, 60(5), B30.

Dohaney, J., De Roiste, M., Salmon, R.A., \& Sutherland, K. (2020). Benefits, barriers, and incentives for improved resilience to disruption in university teaching. International of Disaster Risk Reduction, 50(101691), 1-9.

Fei, M., \& Yeung, D.Y. (2015). Temporal models for predicting student dropout in massive open online courses. In 2015 IEEE International Conference on Data Mining Workshop (ICDMW) (pp. 256-263). IEEE.

Gui, M. (2019). Il digitale a scuola. Rivoluzione o abbaglio? (pp. 1-248). Bologna: Il Mulino.

Harden, R.M., \& Lilley, P. (2018). The eight roles of the medical teacher. Amsterdam: Elsevier Limited.

Hodges, C., Moore, S., Lockee, B., Trust, T., \& Bond, A. (2020). The difference between emergency remote teaching and online learning. Educause Review, 27. Recuperato da: https://er.educause.edu/articles/2020/3/the-difference-between-emergency-remoteteaching-and-online-learning (30.12.20).

Laurillard, D. (2013). Teaching as a design science: Building pedagogical patterns for learning and technology. London: Routledge.

Limone, P., Dipace, A., \& Martiniello, L. (2016). Insegnanti e media digitali. Fattori socio-cognitivi e motivazionali che riducono le resistenze all'innovazione. Pedagogia oggi, 2(2016), 248-257.

Miller, G., Benke, M., Chaloux, B., Ragan, L.C., Schroeder, R., Smutz, W., \& Swan, K. (2014). Leading the e-learning transformation of Higher Education: Meetingthe challenges of technology and distance education. Sterling, VA: Stylus Publishing.

Moreno-Marcos, P.M., Muñoz-Merino, P.J., Maldonado-Mahauad, J., Pérez-Sanagustín, M., Alario-Hoyos, C., \& Kloos, C.D. (2020). Temporal analysis for dropout 
prediction using self-regulated learning strategies in self-paced MOOCs. Computers \& Education, 145(103728), 1-15.

Nikendei, C., Ben-David, M. F., Mennin, S., \& Huwendiek, S. (2016). Medical educators: How they define themselves-Results of an international web survey. Medical teacher, 38(7), 715-723.

Salmon, G. (2014). Learning innovation: A framework for transformation. European Journal of Open, Distance and E-learning, 17(2), 220-236.

Scarinci, A., \& Dipace, A. (2019). Formazione pedagogica per l'insegnamento: il nuovo profilo professionale del docente universitario. In A. Dipace, V. Tamborra (a cura di). Insegnare in Università. Metodi e strumenti per una didattica efficace ( $\mathrm{p}$. 19-36). Milano: Franco Angeli.

Xing, W., Chen, X., Stein, J., \& Marcinkowski, M. (2016). Temporal predication of dropouts in MOOCs: Reaching the low hanging fruit through stacking generalization. Computers in human behaviour, 58, 119-129. 